\title{
Liter per Second
}

National Cancer Institute

\section{Source}

National Cancer Institute. Liter per Second. NCI Thesaurus. Code C67390.

A metric unit of volumetric flow rate defined as the rate at which one liter of matter crosses a given surface during the period of time equal to one second. 